\title{
Chemin de cristallisation informatisé : analyse critique des données
}

\author{
J.A. Musso ${ }^{1}$, H. Hammi ${ }^{2}$, A. M'nif ${ }^{2}$ et R. Rokbani ${ }^{2}$ \\ ${ }^{1}$ Laboratoire de Matériaux et Microélectronique de Provence, Université du Sud-Toulon-Var, \\ BP. 20132, 83957 La Garde Cedex, France \\ 2 Laboratoire des Procédés Chimiques, Institut National de Recherche Scientifique et \\ Technique, BP. 95, 2050 Hamman-Lif, Tunisie
}

\begin{abstract}
In former works we described the DPAO method "Diagram of Phases Computer-assisted" and we applied it to the study of the isothermal and isobar evaporation of a point mixture representing the natural brine resulting from Sebkha El Melah de Zarzis (Tunisia). The quality of the data, as well in precision as in number, is discussed in this presentation, whatever the method of adopted advance: computerized or manual.
\end{abstract}

\section{INTRODUCTION}

Dans des travaux antérieurs [1,2] nous avons décrit la méthode de DPAO « Diagramme de Phases Assisté par Ordinateur » et nous l'avons appliquée à l'étude de l'évaporation isotherme et isobare d'un point mélange représentant la saumure naturelle issue de Sebkha El Melah de Zarzis (Tunisie). La qualité des données, tant en précision qu'en nombre, est discutée dans cette présentation, quelle que soit la méthode de cheminement adoptée : informatisée ou manuelle.

\section{CHEMINEMENTS}

\subsection{Cheminement expérimental}

Nous avons collecté et caractérisé par DRX les différents sels obtenus lors de l'évaporation d'une saumure naturelle prélevée de Aïn Serab de Sebkha El Melah de Zarzis à $298 \mathrm{~K}$ sous la pression de 1 bar. La saumure est assimilée au système quinaire réciproque de classe $\mathrm{II} \mathrm{K}^{+}, \mathrm{Mg}^{2+}, \mathrm{Na}^{+}, \mathrm{Cl}^{-}, \mathrm{SO}_{4}{ }^{2-} / / \mathrm{H}_{2} \mathrm{O}$ saturé en $\mathrm{NaCl}$. La composition de cette saumure, en g.L ${ }^{-1}$, est reportée dans la table 1 [3].

Tableau 1. Concentration, en g.L $\mathrm{L}^{-1}$, de la saumure naturelle prélevée dans le sud tunisien à 298K.

\begin{tabular}{cccccc}
\hline $\mathrm{Mg}^{2+}$ & $\mathrm{Na}^{+}$ & $\mathrm{K}^{+}$ & $\mathrm{Cl}^{-}$ & $\mathrm{SO}_{4}{ }^{2-}$ & densité \\
\hline 53,95 & 41,32 & 7,36 & 215,14 & 30,50 & 1,241 \\
\hline
\end{tabular}

Le résultat de ce cheminement a été discuté aux XXIXièmes JEEP [3]. 


\subsection{Cheminement DPAO}

Du point de vue numérique, un cheminement est un enchaînement logique de procédures (déplacement dans le domaine des liquides non saturés, déplacement dans un domaine de co-cristallisation, bilans de matière, ...) et d'aiguillages (franchissement ou non d'une ligne de co-cristallisation, déshydratation d'un sel, arrêt du cheminement par épuisement du liquide ou atteinte du point de plus basse cote en eau, ...). L'évaporation isotherme et isobare est contrôlée à chaque étape par la nature de la réaction chimique qui peut avoir lieu dans chaque domaine de variance [1]

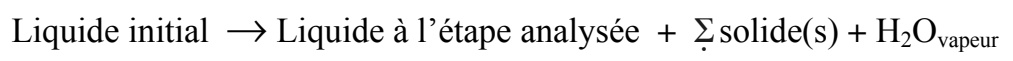

et par l'analyse des bilans de matière.

La figure 1 montre l'écran type d'une représentation particulière (les concentrations des cations en abscisses, celles des anions en ordonnées permettant la visualisation de la relation d'électroneutralité) de l'évaporation de la saumure précédente (Table 1).

La procédure bilan de matière appelle une précision :

D'une manière générale, pour une réaction chimique quelconque, on désigne par $\mathrm{q}_{0}$ la quantité initiale de liquide non saturé dont la composition est le vecteur L, Q le vecteur des quantités de phases, $\mathrm{P}$ la matrice dont les colonnes sont les coefficients normés dans les formules chimiques des produits de la réaction :

$$
\mathrm{q}_{0} \cdot \mathrm{L}=\mathrm{Q} . \mathrm{P}
$$

Le bilan de matière est la procédure qui permet de calculer les éléments du vecteur $\mathrm{Q}$; les éléments de la matrice $\mathrm{P}$ étant connus. Nous appliquons la méthode des moindres carrés afin de prendre en compte les incertitudes dans les compositions des liquides, en particulier et le surdimensionnement du système d'équations suivant l'ordre du système d'équilibres. Ainsi on remplace le système (1) par le système (2):

$$
\left|\mathrm{q}_{0} . \mathrm{L}-\mathrm{Q} . \mathrm{P}\right|^{2} \text { minimum }
$$

La figure 1 est commentée dans le paragraphe suivant.

\section{QUANTITÉ ET QUALITÉ DES DONNÉES}

\subsection{Qualité des données}

Il se dégage de la figure 1 un fait remarquable, malgré la complexité apparente du dessin. La partie supérieure à droite de la figure est un segment de droite qui illustre la relation d'électroneutralité de la solution (à cet effet des espèces, faussement dimères, ont été introduites : $\mathrm{Na}_{2}{ }^{2+}, \mathrm{K}_{2}{ }^{2+}$ et $\mathrm{Cl}_{2}{ }^{2-}$ ). Le tracé symbolisant le cheminement au cours de l'évaporation s'éloigne significativement de ce segment (première séquence du cheminement : déplacement dans le volume de cristallisation de $\mathrm{NaCl}$ ): cela est un moyen de vérifier la cohérence des données. Le diagramme est établi avec les seuls ions $\mathrm{Na}^{+}, \mathrm{Mg}^{2+}, \mathrm{K}^{+}, \mathrm{Cl}^{-}$et $\mathrm{SO}_{4}{ }_{4}^{2-}$, considérés comme ions majeurs de la solution. D'autres ions n'ont pas pu être pris en considération (en particulier $\mathrm{Ca}^{2+}$ et $\mathrm{Br}^{-}$) faute de données 
de solubilité expérimentales suffisantes : la solution initiale n'est pas électriquement neutre : le déficit en charges positives par rapport aux charges négatives est de l'ordre de $5 \%$.

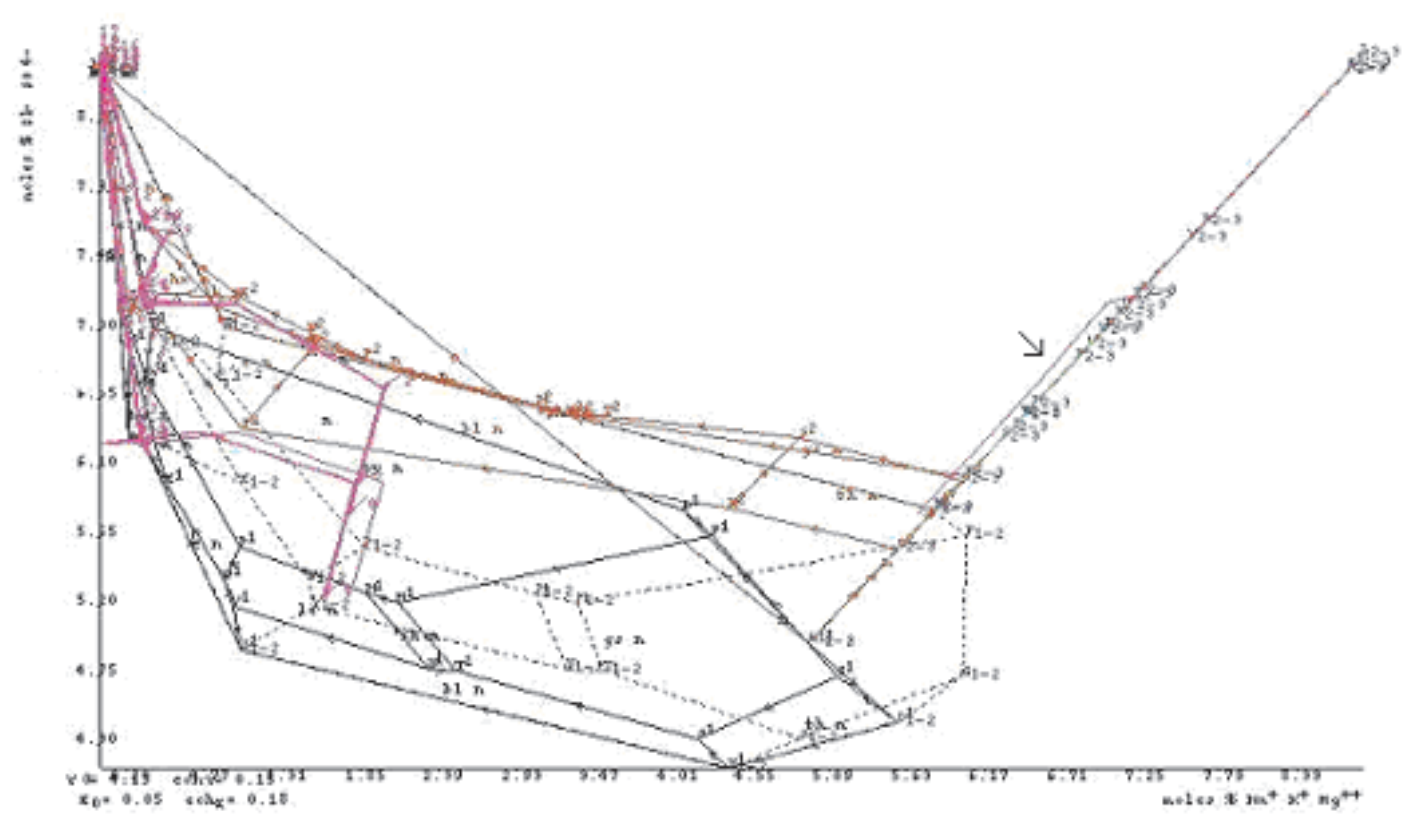

Figure 1. Cheminement obtenu par la méthode de DPAO.

\subsection{Quantité de données}

Le cheminement dans la méthode DPAO repose sur la seule modélisation géométrique du diagramme de phases: l'interpolation linéaire entre deux points. Ce paragraphe montre l'importance qu'il y a à avoir un nombre suffisant de données de solubilité fiables. L'exemple retenu est le soussystème quaternaire réciproque de classe $\mathrm{I} \mathrm{Mg}^{2+}, \mathrm{Na}^{+}, \mathrm{Cl}^{-}, \mathrm{SO}_{4}{ }^{2-} / / \mathrm{H}_{2} \mathrm{O}$ à $298 \mathrm{~K}$ et sous la pression de 1 bar et, plus particulièrement, dans la première étape de l'évaporation : le déplacement dans le domaine des liquides non saturés jusqu'à ce que la nappe de cristallisation de la mirabilite $\mathrm{Na}_{2} \mathrm{SO}_{4} \cdot 10 \mathrm{H}_{2} \mathrm{O}$ soit atteinte.

Pour démontrer l'influence du nombre de points utilisés pour décrire une nappe de cristallisation, nous avons pris quatre descriptions possibles de cette nappe (figure 2).

\subsubsection{Les modèles}

a) La représentation simple avec les seuls points invariants. La nappe s'appuie sur les quatre points invariants :

$\mathrm{B}$ : liquide saturé en mirabilite et $\mathrm{NaCl}$ dans le sous-système $\mathrm{Na}^{+}, \mathrm{SO}_{4}{ }^{2-} / / \mathrm{H}_{2} \mathrm{O}$

$\mathrm{S}$ : liquide saturé en mirabilite, thénardite $\left(\mathrm{Na}_{2} \mathrm{SO}_{4}\right)$ et $\mathrm{NaCl}$ dans le sous-système $\mathrm{Na}^{+}, \mathrm{Cl}^{-}$, $\mathrm{SO}_{4}^{2-} / / \mathrm{H}_{2} \mathrm{O}$ 
Q : liquide saturé en mirabilite, blœdite $\left(\mathrm{MgNa}_{2}\left(\mathrm{SO}_{4}\right)_{2} \cdot 4 \mathrm{H}_{2} \mathrm{O}\right.$ et $\mathrm{NaCl}$ dans le sous-système $\mathrm{Mg}^{2+}, \mathrm{Na}^{+}, \mathrm{SO}_{4}{ }^{2-} / / \mathrm{H}_{2} \mathrm{O}$

$\mathrm{V}$ : liquide saturé en mirabilite, thénardite, blœdite et $\mathrm{NaCl}$

Ces quatre points sont les limites des lignes monovariantes ; B-Q, Q-V, V-S et S-B.

b) Des points monovariants sont ajoutés sur les lignes monovariantes pour en simuler la courbure. Trois points monovariants précisent les courbures des lignes monovariantes..

I (sur la ligne BQ): liquide saturé en mirabilite et $\mathrm{NaCl}$ dans le sous-système $\mathrm{Mg}^{2+}, \mathrm{Na}^{+}$, $\mathrm{SO}_{4}{ }^{2-} / / \mathrm{H}_{2} \mathrm{O}$

$\mathrm{J}$ (sur la ligne QV): liquide saturé en mirabilite, blødite et $\mathrm{NaCl}$

$\mathrm{K}$ (sur la ligne BS): liquide saturé en mirabilite et $\mathrm{NaCl}$ dans le sous-système $\mathrm{Na}^{+}, \mathrm{Cl}^{-}$, $\mathrm{SO}_{4}{ }^{2-} / / \mathrm{H}_{2} \mathrm{O}$

c) La décomposition de la nappe de cristallisation en «facettes », par analogie à la représentation d'une surface à l'aide d'éléments finis, sans point monovariant intermédiaire. Le domaine de cristallisation de la mirabilite dans ce sous-système est représenté par deux facettes triangulaires. Deux possibilités s'offrent si on ne prend en compte que les points invariants : la décomposition avec les deux facettes B-Q-V-B, et B-V-S-B ou la décomposition avec les deux facettes B-Q-S-B et Q-S-V-Q.

Remarque : Les lignes B-V et Q-S définissent des « concavités/convexité » de la nappe. L'allure de la nappe pourrait être affinée avec un point commun à ces deux pliures, point bivariant qui caractérise la concavité ou la convexité de la nappe. La littérature ne renseigne pas sur la composition d'un tel point dans ce système.

d) La décomposition de la nappe de cristallisation en facettes avec les points monovariants intermédiaires. Le domaine de cristallisation de la mirabilite dans ce sous-système est représenté par cinq «facettes» triangulaires s'appuyant sur le point B de plus haute cote en eau. Cette décomposition en pseudo-éléments finis correspond au nombre maximum de facettes qu'il est possible d'obtenir avec les points pris en compte. Les cinq facettes sont : B-I-Q-B, B-Q-J-B, B-J-V-B, B-V-S-B et B-S-K-B.

\subsubsection{Résultats et discussion}

La comparaison sera établie à partir du bilan de matière de la réaction :

100 moles de liquide initial $\rightarrow(100-q-\varepsilon)$ moles de liquide saturé ${ }^{*}+\varepsilon$ moles de mirabilite + $q$ moles de $\mathrm{H}_{2} \mathrm{O}_{\text {vapeur }}$

et du paramètre écart qui est la plus petite distance (en unité de composition) entre un point mélange et la nappe de cristallisation ${ }^{\dagger}$. (table 2 )

\footnotetext{
* On appelle ici «liquide saturé » le liquide au tout début de sa saturation, c'est-à-dire pour un dépôt d'une quantité $\varepsilon$ infinitésimale de solide négligeable devant la quantité q.

${ }^{\dagger}$ Cette distance minimum est retenue pour apprécier l'appartenance d'un point à une nappe de cristallisation.
} 
Figure 2. Représentation, en coordonnées de Jänecke, de la nappe de cristallisation de la mirabilite dans le système réciproque :

$\mathrm{Mg}^{2+} \mathrm{Na}^{+} \mathrm{Cl}^{-} \mathrm{SO}_{4}{ }^{2-} / / \mathrm{H}_{2} \mathrm{O}$ à la température de $298 \mathrm{~K}$ sous la pression de 1 bar.

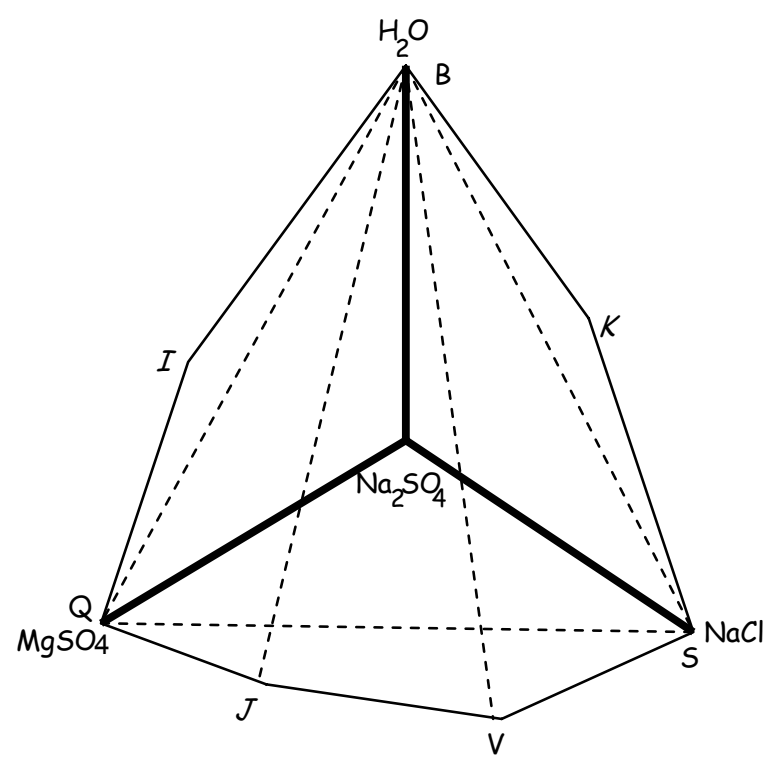

Tableau 2. Cheminement dans le volume de cristallisation de $\mathrm{NaCl}$ en fonction du modèle choisi pour représenter la nappe de cristallisation.

\begin{tabular}{lccc}
\hline \multicolumn{1}{c}{ modèle } & surface atteinte & écart & $\mathrm{q}\left({\left.\text { moles } \mathrm{H}_{2} \mathrm{O}\right)}^{1}\right.$ \\
\hline a) invariants : & B-Q-V-S-B & $0,78 \cdot 10^{-4}$ & 7,14 \\
b) invariants et trois courbures & B-I-Q-J-V-S-K-B & $0,53 \cdot 10^{-5}$ & 7,13 \\
$\begin{array}{l}\text { c) invariants et deux facettes : } \\
\text { facettes B-Q-V-B et B-V-S-B }\end{array}$ & B-Q-V-B & $0,29.10^{-5}$ & 13,72 \\
facettes B-Q-S-B et Q-V-S-Q & B-Q-S-B & $0,22 \cdot 10^{-6}$ & 13,71 \\
d) invariants et cinq facettes & B-J-V-B & $0,12 \cdot 10^{-6}$ & 13,72 \\
\hline
\end{tabular}

Le tableau montre que l'approche d'une nappe de cristallisation est d'autant meilleure que la nappe est décrite avec le plus d'éléments possibles. Ce critère n'a pas de valeur absolue : les cheminements doivent être simulés et confrontés à l'expérience avec toutes les représentations possibles dès lors qu'il s'agit de modèles. Le tableau montre aussi que la quantité d'eau évaporée dépend de l'approche sur la nappe. Les bilans de matière intermédiaires en dépendent aussi. L'incertitude sur le bilan de matière en fin de cheminement peut être substantiellement diminuée si l'on effectue le bilan de matière suivant la réaction (les produits de la réaction à l'étape $\mathrm{n}$ du cheminement deviennent les réactifs de la réaction à l'étape $\mathrm{n}+1 \mathrm{du}$ cheminement):

100 moles de liquide initial $\rightarrow\left(100-q-\Sigma \mathrm{q}_{\mathrm{i}}\right)$ moles de liquide final $+\Sigma \mathrm{q}_{\mathrm{i}}$ moles de solides $+q$ moles de $\mathrm{H}_{2} \mathrm{O}_{\text {vapeur }}$. 


\section{CONCLUSION}

La méthode DPAO a été présentée comme une variante informatisée de l'exploitation manuelle des diagrammes de phases, quel que soit leur ordre. La fiabilité des résultats dépend de la cohérence des données. Elle offre, en outre, l'avantage de pouvoir simuler l'évaporation isotherme et isobare, ou tout autre variation de composition, évitant ainsi des manipulations inutiles, sans pour autant les supprimer totalement.

\section{Références}

[1] Musso J.A.: Solubility Phase Diagrams Coupled to Computer Science (DPAO) Part I : Theory of the Sequential Representation; CALPHAD, 27,1 (2003) 65-69.

[2] Hammi H., Musso J.A., M'nif A., Rokbani R., Solubility Phase Diagrams Coupled to Computer Science (DPAO) Part II : Applied to Isothermal Evaporation of Tunisian Natural Brines; CALPHAD, 27,1 (2003) 71-77.

[3] Hammi H., Musso J.A., M’Nif A. et Rokbani R., Evaporation isotherme et isobare des saumures du sud Tunisien suivie par la méthode DPAO. J. Phys. IV France 113 (2004) 119-123. 